\title{
Treatment of Streptococcal Endocarditis with a Single Daily Dose of Ceftriaxone and Netilmicin for 14 Days: A Prospective Multicenter Study
}

\author{
P. Francioli, W. Ruch, D. Stamboulian, and the \\ International Infective Endocarditis Study Group*
}

From the Centre Hospitalier Universitaire Vaudois, Lausanne;
Hoffmann-La Roche, Basel, Switzerland; and Sanatorio Güemes,
Buenos Aires, Argentina

A 2-week course of ceftriaxone $(2 \mathrm{~g})$ plus netilmicin $(4 \mathrm{mg} / \mathrm{kg})$, administered as one short daily iv infusion, was evaluated for the treatment of streptococcal endocarditis in an open multicenter study. Of the 52 patients, 31 were infected with viridans streptococci, 18 with Streptococcus bovis, two with Gemella morbillorum, and one with group C Streptococcus; 48 patients were assessable. Infection was cured in 42 cases, 35 treated medically and seven treated both medically and surgically. Five patients died without evidence of active infection, and one relapsed. The bacteriologic failure was due to a strain of $G$. morbillorum against which no synergy of ceftriaxone and netilmicin was evident in vitro. The serum creatinine level increased during treatment in four cases, all involving patients $>65$ years old who had renal risk factors; in two of these cases, values did not return to baseline during follow-up. Of $\mathbf{4 0}$ patients assessed for auditory function, only one developed decreased perception of borderline significance. Other adverse reactions were mild. This regimen was efficacious, safe, and cost-effective for the treatment of streptococcal endocarditis. However, it must be used with caution for patients with preexisting renal impairment or concomitant exposure to other potentially nephrotoxic agents.

Four weeks of treatment with iv penicillin alone or 2 weeks of treatment with iv penicillin plus an aminoglycoside are effective for streptococcal endocarditis [1, 2]. However, such regimens require several daily injections, and the use of aminoglycosides carries a risk of renal toxicity and ototoxicity $[2,3]$. Two recent developments may allow more convenient and safer treatment of this condition. First, a single daily dose of ceftriaxone (a cephalosporin with a prolonged serum half-life) given for 4 weeks has been found to be effective against streptococcal endocarditis $[4,5]$. This regimen has been used successfully on an outpatient basis and without a permanent iv catheter. Second, recent experimental and clinical evidence has suggested that administration of the total daily dose of an aminoglycoside in a single injection is as effective as administration of the same amount of drug in two or three injections and causes equal or less toxicity [6-9]. Indeed, in the treatment of experimental streptococcal endocarditis, when given in a single daily dose rather than as multiple doses, aminoglycosides were just as synergistic with $\beta$-lactam agents such as penicillin and ceftriaxone [10-13].

The purpose of the multicenter study reported herein was to test prospectively the efficacy and safety of a 2 -week course of

Received 24 April 1995; revised 13 July 1995.

Reprints or correspondence: Dr. P. Francioli, Division Autonome de Médecine Préventive Hospitalière, Centre Hospitalier Universitaire Vaudois, 1011 Lausanne, Switzerland.

*Study group members are listed after the text.

Clinical Infectious Diseases 1995;21:1406-10

(C) 1995 by The University of Chicago. All rights reserved.

$1058-4838 / 95 / 2106-0019 \$ 02.00$ therapy for streptococcal (nonenterococcal) endocarditis with a regimen consisting of ceftriaxone plus netilmicin given once a day. The study was noncomparative since streptococcal endocarditis is a rare disease and the outcome of treatment with penicillin is well known. The efficacy of penicillin is so high that it would have been impossible to recruit enough patients to exclude a type II error.

\section{Patients and Methods}

Adults with ceftriaxone-susceptible streptococcal nativevalve endocarditis were eligible for the study. The diagnostic criteria for endocarditis were persistent bacteremia (i.e., at least three sets of positive blood cultures, with samples drawn at intervals of at least 30 minutes) plus (1) a new or changing heart-valve regurgitation murmur and/or (2) a predisposing heart disease associated with vascular phenomena and/or with cardiac vegetations visualized by transthoracic echocardiography [14]. The newly described criteria for the diagnosis of endocarditis were also applied retrospectively [15].

Patients were excluded if they met any of the following criteria: (1) antibiotic treatment for $>48$ hours before enrollment; (2) hypersensitivity to cephalosporins or aminoglycosides; (3) impairment of renal function, with serum creatinine values at least $30 \%$ above the normal limit; or (4) clinically apparent inner-ear or vestibular dysfunction. Ceftriaxone sodium and netilmicin were administered for 14 days at daily doses of $2 \mathrm{~g}$ and $4 \mathrm{mg} / \mathrm{kg}$, respectively. Ceftriaxone was diluted in $50 \mathrm{~mL}$ of $5 \%$ glucose and netilmicin in a similar volume of $0.9 \% \mathrm{NaCl}$. Both drugs were infused iv; the infusions lasted 30 and 60 minutes, respectively. 
Microbiological tests included cultures of blood and of other potentially infected sites. Bacterial isolates were identified with the API 20 Strep System (bioMérieux, Marcy l'Étoile, France). Antibiotic susceptibility was assessed both by the Kirby-Bauer method and by the determination of MICs with a standard macrotube dilution technique.

Patients were evaluated daily during hospitalization as well as once at the end of treatment, once between 2 and 4 weeks after the completion of treatment, and once between 2 and 3 months after the end of therapy. Hematologic and chemical analyses of blood and blood cultures, as well as urinalysis, were undertaken before, during, and after treatment. Echocardiography and audiometry were performed at the beginning and end of treatment. Serum levels of netilmicin were determined twice a week during treatment, both immediately before and 30 minutes after the end of infusion.

An infection was considered clinically cured if the patient had no signs or symptoms suggestive of infection 3 months after the completion of therapy. Bacteriologic cure was defined by sterile blood cultures 3 months after therapy or by sterile cardiac valves at surgery or autopsy.

Informed consent was obtained in all cases. The study was approved by the ethical committees of the participating centers.

\section{Results}

A total of 52 patients (11 females and 41 males) were included in the study. When the new criteria for the diagnosis of endocarditis were applied retrospectively (and, in most cases, without transesophageal echocardiography), 47 patients were categorized as definite cases and five as probable cases. The patients' median age was 61 years (range, $18-80$ years). Thirty patients had a known underlying valvular or congenital cardiac abnormality. Mitral-valve infection was documented in 23 cases $(44 \%)$; aortic infection in 18 cases $(35 \%)$; combined mitral and aortic infection in 10 cases (19\%); and combined mitral, aortic, and tricuspid infection in one case ( $2 \%$ ). Twentyone patients had definite vegetations visualized by echocardiography.

The bacteria isolated from blood were Streptococcus bovis in 18 cases $(35 \%)$, Streptococcus sanguis in 14 cases $(27 \%)$, Streptococcus mutans in six cases $(12 \%)$, nongroupable viridans streptococci in five cases $(10 \%)$, Streptococcus milleri and Streptococcus mitis in three cases each $(6 \%)$, Gemella morbillorum in two cases (4\%), and group C Streptococcus in one case $(2 \%)$. None of the patients had another potential source of persistent bacteremia. All strains were susceptible to penicillin and ceftriaxone in the disk diffusion test. MICs were determined for 47 of the 52 strains. The $\mathrm{MIC}_{90}$ was 0.25 $\mu \mathrm{g} / \mathrm{mL}$ (range, $0.016-0.5 \mu \mathrm{g} / \mathrm{mL}$ ) for ceftriaxone and penicillin and $2 \mu \mathrm{g} / \mathrm{mL}$ (range, $0.03-8 \mu \mathrm{g} / \mathrm{mL}$ ) for netilmicin.

After the drawing of blood cultures, 14 patients $(27 \%)$ had been empirically treated for $24-48$ hours before enrollment
Table 1. Outcome of 52 cases of streptococcal endocarditis treated with a 2-week course of ceftriaxone $(2 \mathrm{~g})$ and netilmicin $(4 \mathrm{mg} / \mathrm{kg}$ ) administered in a single daily dose.

\begin{tabular}{lr} 
Outcome & No. of cases \\
\hline Clinical & \\
Cure & 35 \\
$\quad$ With antibiotic treatment only & 7 \\
$\quad$ With antibiotic treatment and surgery & 1 \\
Relapse & 5 \\
Death* & 2 \\
Premature discontinuation of treatment & 1 \\
Treatment for $>15$ days & 1 \\
Loss to follow-up & 42 \\
Bacteriologic & 2 \\
Cured & 1 \\
Presumed cure & 7 \\
Failure & \\
Unassessable & \\
\hline & * No evidence of active infection. \\
${ }^{\dagger}$ Sterile blood culture at 3 months (35 cases) and/or sterile cardiac valves \\
at surgery or autopsy ( 7 cases). \\
${ }^{\ddagger}$ No clinical evidence of disease at 3 and 6 months, respectively. \\
${ }^{*}$ Infection due to Gemella morbillorum.
\end{tabular}

with antibiotics unrelated to the study drugs. The regimen was switched to ceftriaxone plus netilmicin as soon as the results of blood culture became available.

Table 1 summarizes the clinical and bacteriologic outcome of the 52 cases. Four patients ( $8 \%$ ) could not be fully assessed for the following reasons. The first completed treatment but did not return for follow-up and could not be located. The second received ceftriaxone for a total of 35 days because of a suspected concomitant hip-joint infection; his infection was cured clinically and bacteriologically. The third and fourth patients discontinued treatment prematurely: the regimen was changed to penicillin because of worsening renal function in one case (on day 4) and to penicillin and gentamicin because of the suspicion of rheumatic fever in the other (on day 8).

of the 48 clinically assessable cases, 42 were cured - 35 with antibiotic treatment alone and seven with combined antibiotic and surgical therapy. One patient relapsed (see below). Five patients died. Two of the deaths occurred within a few hours after valve replacement (on days 14 and 27, respectively), and in these cases the valves as well as the blood cultured on the same day were sterile. Another two deaths (on days 25 and 26 , respectively) were related to the progression of heart failure due to valvular insufficiency that could not be alleviated by surgery; both of the patients involved already had signs of heart failure while receiving antibiotic treatment. No autopsy was conducted in these two cases, but no clinical evidence of active infection was found and cultures of blood obtained on days 16 and 19 , respectively, were sterile. The fifth death occurred suddenly on day 62,36 hours after a colostomy performed for the resection of an adenoma; no autopsy was performed. In 
this case the last blood sample cultured was obtained on day 22 and was sterile. The patient had no signs or symptoms of active infection during the follow-up period.

A total of 43 cases were fully assessable bacteriologically; infection was cured in 42 cases (98\%). Two additional infections were considered presumably cured bacteriologically (according to recent guidelines for trials of antibiotic treatment for endocarditis [14]) since the patients were well at 3 and 6 months, respectively. However, these patients had moved abroad and could not return for follow-up blood cultures.

One relapse was documented in a 49-year-old male. Two months before admission, he had undergone dental treatment. Two weeks later, he had developed fever without other symptoms. At the time of his admission, two-dimensional echocardiography disclosed mitral-valve prolapse without insufficiency and no visible vegetations. Blood cultures yielded a Streptococcus-like microorganism that was later identified as G. morbillorum. Three weeks after the completion of an uneventful course of antibiotic treatment lasting 15 days, fever recurred and blood cultures yielded the same microorganism. For both strains, the MIC of ceftriaxone was $0.25 \mu \mathrm{g} / \mathrm{mL}$ and that of netilmicin was $0.5 \mu \mathrm{g} / \mathrm{mL}$. The patient was successfully treated with a 4-week course of antibiotics: ceftriaxone (days 1-14), amoxicillin (days 15-28), and netilmicin (days 1-20). Timekill kinetics evaluated later showed tolerance to ceftriaxone and an absence of synergy between ceftriaxone and netilmicin.

Nine patients underwent a total of 12 valve replacements either during the treatment period (days 11,13 , and 14, respectively) or during follow-up. The indication for surgery in all cases was valvular incompetence. In seven cases the valves were cultured and were sterile. In the two cases in which valves were not cultured, cultures of blood obtained during followup and before valve replacement were sterile.

A total of 19 adverse events related or possibly related to antibiotic treatment were documented in 18 cases $(35 \%)$. The serum creatinine level increased during therapy in four cases, all involving patients $>65$ years old; baseline values had been elevated in two of these cases. In all four cases at least one other contributing factor was identified (congestive cardiac failure in two cases, diabetes in one case, hypertension in two cases, and concomitant treatment with another nephrotoxic medication in three cases), and in one case an overdose of netilmicin was inadvertently administered. The dosage of netilmicin was adjusted in all four cases.

During follow-up, the serum creatinine level remained elevated in two cases and returned to near-baseline values in the other two. A slightly abnormal creatinine value was noted on a single occasion during the follow-up period in two additional instances. A transient twofold increase in transaminase values was documented in three cases and candidal stomatitis in one case. Five patients developed transient adverse reactions that resolved without the interruption of therapy: there was one case each of diarrhea (a 2-day episode), macular rash, flushing of the face during drug infusion, shivering during drug infusion, and pruritus. Forty patients had audiometry performed at baseline and at the end of treatment; a 27-year-old male had a unilateral 20-decibel (15\%) decrease in both aerial and osseous perception of high-frequency sounds. (A decrease of $>15 \%$ is considered significant.) Three patients developed catheterrelated phlebitis.

The peak and trough levels (mean $\pm \mathrm{SD}$ ) of netilmicin in serum were $10.57 \pm 4.59 \mu \mathrm{g} / \mathrm{mL}$ and $0.89 \pm 0.76 \mu \mathrm{g} / \mathrm{mL}$, respectively. No drug accumulation was detected during treatment.

Twenty-five patients were treated without indwelling catheters. Antibiotics were administered daily to these patients through the placement of a butterfly needle. Twenty patients received the antibiotics through a small peripheral catheter, with or without a heparin lock between infusions. Three patients had a central venous line, and the mode of administration was not recorded for four cases. Five patients were treated solely as outpatients and 10 as both inpatients and outpatients. Of the total of 672 days of antibiotic therapy for the 48 assessable patients, 124 were outpatient days.

\section{Discussion}

In the present study, we tested the efficacy and safety of once-daily administration of ceftriaxone plus netilmicin in the treatment of streptococcal endocarditis. This regimen was selected because of the pharmacokinetic properties of ceftriaxone [16] and the recent suggestions that a single daily injection of an aminoglycoside is just as efficacious as and possibly less toxic than multiple injections of the same total daily dose [613]. Of 48 assessable patients, 42 had their infections cured, five died during the follow-up period (none with evidence of active infection), and one relapsed.

Although this study was not comparative, the relapse rate was not different from those reported after treatment with penicillin or ceftriaxone alone for 4 weeks $[1,4,5]$ or with penicillin plus streptomycin for 2 weeks $[2,3]$. The relapse was due to a microorganism of a genus (Gemella) that is now recognized as separate from Streptococcus and that has been implicated in complicated endocarditis [4]. No synergy of ceftriaxone and netilmicin against this organism was apparent in vitro, although synergy between $\beta$-lactam antibiotics and aminoglycosides is observed with most if not all streptococcal strains [17].

The mortality rate among the patients in this study $(10 \%)$ is not unexpected in viridans streptococcal endocarditis [1], although rates have been lower in some recent studies $[2,4$, 5]. Deaths were due to several causes: two were related to immediate postoperative complications (in patients whose valves were sterile), one occurred suddenly at 2 months in a patient with no evidence of active infection, and two were due to progressive heart failure (cardiac surgery was postponed for nonmedical reasons). Although active infection was not 
excluded in the last two cases by culture at autopsy, the patients responded clinically to antibiotic treatment, and blood cultures were sterile 1 and 4 days after treatment-that is, 1 week before death. Thus, although uncontrolled infection cannot be totally ruled out in some fatal cases, it appears unlikely.

The most frequent and severe adverse event was an increase in the creatinine serum level, which was recorded in four cases $(8 \%)$ during treatment. All four patients involved were elderly and had other characteristics that may have contributed to renal impairment. In two of these cases, creatinine values remained elevated during the follow-up period. The implication is that, in patients with renal risk factors, an antibiotic regimen that does not include an aminoglycoside must be preferred. In a prior study of 59 patients treated with ceftriaxone alone, none experienced a deterioration of renal function during therapy [4]. However, a 4-week course of monotherapy with $\beta$-lactam antibiotics is complicated by other, potentially severe side effects, such as neutropenia $[4,18]$.

Two aspects of our findings are of special interest. First, a single daily injection of an aminoglycoside combined with ceftriaxone was bactericidal to a degree that permitted a short (2-week) course of therapy for streptococcal endocarditis. Although it is not known whether ceftriaxone alone would yield comparable results, penicillin administered alone for 2 weeks has resulted in failure rates as high as $15 \%$ [19]. Since penicillin and ceftriaxone exhibit similar in vitro activity against streptococci [4], it is likely that in vivo synergy accounts for the high efficacy of the regimen used in the present study. It is remarkable that these results were obtained despite the fact that the single daily injection of the aminoglycoside did not produce therapeutic levels of this drug in serum for 24 hours in most cases. In this study we used netilmicin $(4 \mathrm{mg} /[\mathrm{kg} \cdot \mathrm{d}])$, but experimental and clinical studies suggest that the results would be similar with gentamicin or tobramycin $(3 \mathrm{mg} /[\mathrm{kg} \cdot \mathrm{d}])[6,8$, $11,13]$.

In studies of experimental streptococcal endocarditis, a single daily dose of an aminoglycoside in combination with penicillin or ceftriaxone resulted in a degree of in vivo synergy equal to that attained with multiple injections of the same total daily dose [10-13]. Several clinical studies compared oncedaily to twice- or thrice-daily aminoglycoside administration, mostly in combination with $\beta$-lactam antibiotics; the two regimens were equally efficacious [6-9]. However, none of these studies included patients with infections for which the superiority of a combined regimen is as well established as it is in endocarditis. Thus, the present study adds to the evidence that the constant, simultaneous presence of therapeutic levels of aminoglycosides and $\beta$-lactam antibiotics is not required for in vivo synergy against streptococci. This finding may also apply to other bacteria but may not hold true for all and is especially unlikely to extend to enterococci $[20,21]$.

Second, the antibiotic regimen administered in our study represents a simplification compared with traditional treatment.
For many patients with streptococcal endocarditis, the need for several daily injections of parenteral antibiotics is the main reason for prolonged hospitalization, and recent efforts to improve therapy have been directed at a shorter course (e.g., high doses of penicillin combined with two or three daily injections of an aminoglycoside for 2 weeks) [3] or at the administration of the total daily dose in only one injection, obviating the need for a permanent iv line and allowing partial or complete outpatient treatment in some cases $[4,5]$. These approaches are not only more comfortable for the patient but also less expensive [22]. Outpatient treatment of endocarditis is already used in practice by many physicians [23]. In two studies of therapy with ceftriaxone alone for 4 weeks or with ceftriaxone for 2 weeks followed by oral amoxicillin for 2 weeks, outpatient treatment was found to be effective and safe, provided that patients were appropriately selected $[4,5]$. With the regimen used in our study, the burden of treatment can be further reduced, and some patients - once evaluated and stabilized in the hospital - can be sent home and receive only a few additional days of outpatient treatment with one daily injection of antibiotics.

\section{Members of the International Infective Endocarditis Study Group}

J.-C. Auvergnat, France (contributed 2 cases to this study); A. Daum, Switzerland; P. Francioli, Switzerland (8); R.-L. Galeazzi, Switzerland (1); A. Gerber, Switzerland (2); M. Gringberg, Brazil (1); L. Havas, Switzerland; R. Ramos, Brazil (2); E. Rubinstein, Israel (1); W. Ruch, Switzerland; D. Stamboulian, Argentina (28); J.-P. Thys, Belgium (4); and W. Zimmerli, Switzerland (3).

\section{References}

1. Bisno AL, Dismukes WE, Durack DT, et al. Antimicrobial treatment of infective endocarditis due to viridans streptococci, enterococci, and staphylococci. JAMA 1989;261:1471-7.

2. Wilson WR, Thompson RL, Wilkowske CJ, et al. Short-term therapy for streptococcal infective endocarditis. JAMA 1981;245:360-3.

3. Wilson WR. Ceftriaxone sodium therapy of penicillin $\mathrm{G}$-susceptible streptococcal endocarditis. JAMA 1992;267:279-80.

4. Francioli P, Etienne J, Hoigné R, Thys JP, Gerber A. Treatment of streptococcal endocarditis with a single daily dose of ceftriaxone for 4 weeks: efficacy and out-patient treatment feasibility. JAMA 1992;267:264-7.

5. Stamboulian D, Bonvehi P, Arevalo C, et al. Antibiotic management of outpatients with endocarditis due to penicillin-susceptible streptococci. Rev Infect Dis 1991; 13(suppl 2):S160-3.

6. Ter Braak EW, de Vries PJ, Bouter KP, et al. Once-daily dosing regimen for aminoglycoside plus $\beta$-lactam combination therapy of serious bacterial infections: comparative trial with netilmicin plus ceftriaxone. Am $\mathrm{J}$ Med 1990; $89: 58-66$

7. Van der Auwera P, Meunier F, Ibrahim S, Kaufman L, Derde MP, Tulkens PM. Pharmacodynamic parameters of toxicity of netilmicin (6 milligrams/kilogram/day) given once daily or in three divided doses to cancer 
patients with urinary tract infection. Antimicrob Agents Chemother 1991;35:4640 7.

8. Prins JM, Büller HR, Kuijper EJ, Tange RA, Speclman P. Once vs thrice daily gentamicin in patients with serious infections. Lancet 1993; 341:335-9.

9. International Antimicrobial Therapy Cooperative Group of the European Organization for Research and Treatment of Cancer. Efficacy and toxicity of single daily doses of amikacin and ceftriaxone vs multiple daily doses of amikacin and ceftazidime for infection in patients with cancer and granulocytopenia. Ann Intern Med 1993; 119:584-93.

10. Francioli P, Glauser MP. Synergistic activity of ceftriaxone combined with netilmicin administered in one daily dose in the treatment of experimental streptococcal endocarditis. Antimicrob Agents Chemother 1993; $37: 207-12$.

11. Saleh-Mghir A, Crémieux AC, Vallois JM, Muffat-Joly M, Devine C, Carbon C. Optimal aminoglycoside dosing regimen for penicillin-tobramycin synergism in experimental streptococcus adjacent endocarditis. Antimicrob Agents Chemother 1992;36:2403-7.

12. Blatter M, Fluckiger U, Entenza J, Glauser MP, Francioli P. Simulated human serum profiles of one daily dose of ceftriaxone + netilmicin in the treatment of experimental streptococcal endocarditis. Antimicrob Agents Chemother 1993;37:1971-6.

13. Gavalda J, Pahissa A, Almirante B, et al. Efficacy of gentamicin once a day in the treatment of viridans streptococcal experimental endocarditis [abstract no 1449]. In: Program and abstracts of the 32nd Interscience Conference on Antimicrobial Agents and Chemotherapy (Anaheim). Washington, DC: American Society for Microbiology, 1992.
14. Wilson WR, Gilbert DN, Bisno AL, et al. Evaluation of new anti-infective drugs for the treatment of infective endocarditis. Clin Infect Dis 1992; 15(suppl 1):S89-95.

15. Durack DT, Lukes AS, Bright DK, the Duke Endocarditis Service. New criteria for diagnosis of infective endocarditis: utilization of specific echocardiographic findings. Am J Med 1994;96:200-9.

16. Patel IH, Kaplan SA. Pharmacokinetic profile of ceftriaxone. Am J Med 1984; 77:17-25.

17. Shanson DC, Tadayon M, Bakhtiar M. Bactericidal activity of netilmicin compared with gentamicin and streptomycin, alone and in combination with penicillin, against penicillin tolerant viridans streptococci and enterococci. J Antimicrob Chemother 1986; 18:479-90.

18. Olaison $\mathrm{L}$, Alestig K. A prospective study of neutropenia induced by high doses of betalactam antibiotics. J Antimicrob Chemother 1990;25: $449-53$.

19. Wilson WR, Geraci JE. Treatment of streptococcal infective endocarditis. Am J Med 1985; 78(suppl 6B): 128-33.

20. Fantin $B$, Carbon $C$. Importance of the aminoglycoside dosing regimen in the penicillin-netilmicin combination for treatment of Enterococcus faecalis induced experimental endocarditis. Antimicrob Agents Chemother $1990 ; 34: 2387-91$.

21. Hessen MT, Pitsakis PG, Levison ME. Post-antibiotic effect of penicillin plus gentamicin versus Enterococcus faecalis in vitro and in vivo. Antimicrob Agents Chemother 1989;33:608-11.

22. Francioli PB. Ceftriaxone and outpatient treatment of infective endocarditis. Infect Dis Clin North Am 1993; 7:97-115.

23. Patton JP. Economic considerations. In: Kaye D, ed. Infective endocarditis. 2nd ed. New York: Raven Press, 1992:413-22. 